\title{
Reversal of Vitamin K Antagonist Therapy by Prothrombin Complex Concentrate before Thrombolysis for Acute Stroke
}

\author{
Nicolas Chausson Manvel Aghasaryan Djibril Soumah Tony Altarcha \\ Didier Smadja
}

Stroke Unit, Centre Hospitalier Sud Francilien, Corbeil-Essonnes, France

Vitamin K antagonist (VKA) frequently fails to prevent cerebral infarction, with $30 \%$ of VKA-treated patients experiencing ischemic stroke despite an international normalized ratio (INR) of $\geq 2$ [1]. Intravenous (IV) thrombolysis is only recommended for patients with an INR of $<1.7$ [2]. Indeed, experimental results confirmed the high risk of post-thrombolytic hemorrhages at therapeutic INR levels [3]. However, administration of prothrombin complex concentrate (PCC) and vitamin K can obtain rapid and sustained reversal of VKA-induced coagulopathy with a very low $(<1.7 \%)$ thrombotic risk [4]. This reversal strategy was effective in two rodent models of ischemic stroke with prior effective anticoagulation (INR range: $2-3.5)[3,5]$. Only a few isolated human cases of VKA antagonized by recombinant factor VIIa, fresh frozen plasma or PCC preceding IV [6] or intra-arterial thrombolysis have been reported.

Our group is conducting a prospective pilot study to determine whether IV thrombolysis immediately after a 15-min infusion of PCC (and vitamin K) is safe and effective in ischemic stroke patients with magnetic resonance angiography-proven arterial occlusion and an INR of $>1.7$. The mean \pm SD values of the 4 patients included to date are the following: age $82 \pm$ 1.3 years, National Institute of Health Stroke Scale (NIHSS) score of $14 \pm 2.7$, initial INR of 2.1 (range: 1.8-2.4), and stroke onset-to-needle time of $187 \pm 23 \mathrm{~min}$. The mean post-reversal INR declined to 1.23 (range: 1.2-1.3), and no thrombotic complications occurred. The mean 24-hour NIHSS score was $6.5 \pm 3.8$. Of the 4 included patients, 3 were fully recanalized on 24-hour control magnetic resonance imaging, and 1 had an asymptomatic thrombolysisrelated type 1 intracerebral hemorrhage. These preliminary observations are very encouraging. The real therapeutic challenge might be not only extending IV thrombolysis to ischemic stroke patients on VKA, regardless of their INR value, but also using a similar strategy in patients receiving new oral anticoagulants if effective antidotes become available.

\section{Disclosure Statement}

The authors have no conflicts of interest to declare. 
Chausson et al:: Reversal of Vitamin K Antagonist Therapy by Prothrombin Complex Concentrate before Thrombolysis for Acute Stroke

\section{References}

1 Haeusler KG, Konieczny M, Endres M, Villringer A, Heuschmann PU: Impact of anticoagulation before stroke on stroke severity and long-term survival. Int J Stroke 2012;7:544-550.

-2 Xian Y, Liang L, Smith EE, et al: Risks of intracranial hemorrhage among patients with acute ischemic stroke receiving warfarin and treated with intravenous tissue plasminogen activator. JAMA 2012;307:2600-2608.

-3 Pfeilschifter W, Spitzer D, Pfeilschifter J, Steinmetz H, Foerch C: Warfarin anticoagulation exacerbates the risk of hemorrhagic transformation after rt-PA treatment in experimental stroke: therapeutic potential of PCC. PLoS One 2011;6:e26087.

-4 Leissinger CA, Blatt PM, Hoots WK, Ewenstein B: Role of prothrombin complex concentrates in reversing warfarin anticoagulation: a review of the literature. Am J Hematol 2008;83:137-143.

-5 Sun L, Zhou W, Ploen R, Heiland S, Zorn M, Veltkamp R: Rapid reversal of anticoagulation prevents excessive secondary hemorrhage after thrombolysis in a thromboembolic model in rats. Stroke 2011;42:3524-3529.

-6 Jalini S, Jin AY, Taylor SW: Reversal of warfarin anticoagulation with prothrombin complex concentrate before thrombolysis for acute stroke. Cerebrovasc Dis 2012;33:597. 